\title{
MORE ON CONNECTEDNESS IM KLEINEN AND LOCAL CONNECTEDNESS IN $C(X)$
}

\author{
JACK T. GOODYKOONTZ, JR.
}

\begin{abstract}
Let $X$ be a compact connected metric space and $2^{X}(C(X))$ denote the hyperspace of closed subsets (subcontinua) of $X$. Let $M \in$ $C(X)$. If $2^{X}$ is connected im kleinen at $M$, then $C(X)$ is locally arcwise connected at $M$. A characterization of connectedness im kleinen in $C(X)$ is given. Indecomposability of $X$ is related to an absence of local connectedness in $2^{X}$ and $C(X)$. An example is given of a continuum $X$ and a subcontinuum $M$ such that $C(X)$ is connected im kleinen at $M$ but not locally connected at $M$.
\end{abstract}

A continuum will be a compact connected metric space and $X$ will denote a continuum throughout. Each of $2^{X}$ and $C(X)$ is endowed with the finite (Vietoris) topology and, since $X$ is a continuum, each of $2^{X}$ and $C(X)$ is also a continuum (see [4]).

In an earlier paper by the author [1], the properties of local connectedness and connectedness im kleinen were characterized at points in $2^{X}$ in terms of the neighborhood structure of the corresponding sets in $X$. In this paper the internal structure of $C(X)$ is investigated with respect to these properties.

For notational purposes, small letters will denote elements of $X$, capital letters will denote subsets of $X$ and elements of $2^{X}$, and script letters will denote subsets of $2^{X}$. If $A \subset X$, then $A^{*}$ (int $A$ ) (bd $A$ ) will denote the closure (interior) (boundary) of $A$ in $X$.

Let $x \in X$. Then $X$ is connected im kleinen at $x$ if for each open set $U$ containing $x$ there is a component of $U$ which contains $x$ in its interior. $X$ is locally connected (locally arcwise connected) at $x$ if for each open set $U$ containing $x$ there is a connected open set (arcwise connected open set) $V$ such that $x \in V \subset U$.

If $A_{1}, \ldots, A_{n}$ are subsets of $X$, then $N\left(A_{1}, \ldots, A_{n}\right)=\left\{B \in 2^{X} \mid\right.$ for each $i=1, \ldots, n, B \cap A_{i} \neq \varnothing$, and $\left.B \subset \cup_{i=1}^{n} A_{i}\right\}$. An order arc in $2^{X}(C(X))$ is an arc which is also a chain with respect to the partial order on $2^{X}(C(X))$ induced by set inclusion. If $A, B \in 2^{X}(C(X))$, then there exists an order arc in $2^{X}(C(X))$ from $A$ to $B$ if and only if $A \subset B$ and each component of $B$ intersects $A$ (Lemmas 2.3 and 2.6 of [3]).

We begin with a theorem which answers Question 1 of [1] in the affirma-

Received by the editors November 3, 1975 and, in revised form, November 29, 1976.

AMS (MOS) subject classifications (1970). Primary 54B20; Secondary 54D05.

Key words and phrases. Continuum, hyperspace, connected im kleinen, locally connected.

- American Mathematical Society 1977 
tive, and which in fact provides a more general result.

THEOREM 1. Let $M \in C(X)$. If $2^{X}$ is connected im kleinen at $M$, then $C(X)$ is locally arcwise connected at $M$.

Proof. Let $M \in C(X)$ and $N\left(U_{1}, \ldots, U_{n}\right) \cap C(X)$ be a basic open set in $C(X)$ containing $M$. Let $N\left(V_{1}, \ldots, V_{m}\right)$ be an open set such that $M \in$ $N\left(V_{1}, \ldots, V_{m}\right)$ and $N\left(V_{1}, \ldots, V_{m}\right)^{*} \subset N\left(U_{1}, \ldots, U_{n}\right)$. Let $\bigodot$ be the component of $N\left(V_{1}, \ldots, V_{m}\right)$ which contains $M$. Since $2^{X}$ is connected im kleinen at $M, \mathcal{C}$ contains $M$ in its interior, so there exists an open set $N\left(W_{1}, \ldots, W_{p}\right)$ such that $M \in N\left(W_{1}, \ldots, W_{p}\right) \subset \mathcal{C} \subset N\left(V_{1}, \ldots, V_{m}\right)$. Let $C=(\cup \mathcal{C})^{*}$. Then $C \in C(X)$ and $C \in N\left(V_{1}, \ldots, V_{m}\right)^{*}$, so $C \in$ $N\left(U_{1}, \ldots, U_{n}\right)$. Let $U=\cup_{i=1}^{n} U_{i}$ and note that $M, C \in$ $N\left(W_{1}, \ldots, W_{p}, U\right) \subset N\left(U_{1}, \ldots, U_{n}\right)$.

We will now show that $N\left(W_{1}, \ldots, W_{p}, U\right) \cap C(X)$ is arcwise connected. Let $L_{1}, L_{2} \in N\left(W_{1}, \ldots, W_{p}, U\right) \cap C(X), L_{1} \neq L_{2}$. Since $\cup_{i=1}^{p} W_{i} \subset C$, it follows that $L_{1} \cap C \neq \varnothing$ and $L_{2} \cap C \neq \varnothing$. Hence $L_{1} \cup L_{2} \cup C \in$ $N\left(W_{1}, \ldots, W_{p}, U\right) \cup C(X)$. So there exist order arcs $\varrho_{1}$ and $\varrho_{2}$ from $L_{1}$ to $L_{1} \cup L_{2} \cup C$ and from $L_{2}$ to $L_{1} \cup L_{2} \cup C$. It follows that there is an arc in $\mathscr{L}_{1} \cup \mathscr{L}_{2}$ from $L_{1}$ to $L_{2}$, and it is clear that $\mathcal{L}_{1} \cup \mathscr{L}_{2} \subset N\left(W_{1}, \ldots, W_{p}, U\right) \cap$ $C(X)$.

Theorem 2. Let $M \in C(X)$. Then $C(X)$ is connected im kleinen at $M$ if and only if for each open set $U$ containing $M$, each sequence of continua contained in $U$ which converges to $M$ is eventually contained in the component of $U$ to which $M$ belongs.

Proof. Suppose $C(X)$ is connected im kleinen at $M$. Let $U$ be an open set containing $M$ and let $D$ denote the component of $U$ to which $M$ belongs. Let $\left\{L_{i}\right\}_{i=1}^{\infty}$ be a sequence of continua contained in $U$ such that $L_{i} \rightarrow M$. Let $\mathcal{C}$ denote the component of $N(U) \cap C(X)$ to which $M$ belongs. Since $C(X)$ is connected im kleinen at $M$ there exists an open set $N\left(V_{1}, \ldots, V_{n}\right) \cap C(X)$

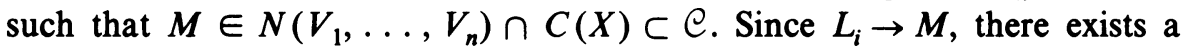
positive integer $i_{0}$ such that $i \geqslant i_{0}$ implies $L_{i} \in N\left(V_{1}, \ldots, V_{n}\right) \cap C(X)$. Since $\mathcal{C}$ is a connected subset of $C(X), \cup\{C \in C(X) \mid C \in \mathcal{C}\}$ is a connected subset of $X$, and $\cup\{C \in C(X) \mid C \in \mathcal{C}\} \subset U$. Then $\cup_{i=i_{0}}^{\infty} L_{i} \subset$ $\cup\{C \in C(X) \mid C \in \mathcal{C}\} \subset D$. So $\left\{L_{i}\right\}_{i=1}^{\infty}$ is eventually contained in $D$.

For the converse, suppose that $C(X)$ is not connected im kleinen at $M$. Then there exists an open set $\mathscr{U} \cap C(X)$ and a sequence $\left\{\bigodot_{i}\right\}_{i=1}^{\infty}$ of distinct components of $(\mathscr{U} \cap C(X))^{*}$ converging to a limit continuum $\mathcal{C}$ which contains $M$ and has no point in common with any of the continua $\mathcal{C}_{i}$ (Theorem 12.1 on p. 18 of [5]). Choose basic open sets $N\left(U_{1}, \ldots, U_{n}\right)$ and $N\left(V_{1}, \ldots, V_{m}\right)$ each containing $M$ such that

$$
\begin{gathered}
\left(N\left(U_{1}, \ldots, U_{n}\right) \cap C(X)\right)^{*} \subset N\left(U_{1}, \ldots, U_{n}\right)^{*} \cap C(X) \\
\subset N\left(V_{1}, \ldots, V_{m}\right) \cap C(X) \subset \mathcal{Q} \cap C(X) .
\end{gathered}
$$


Since $\mathcal{C}_{i} \rightarrow \mathcal{C}$ and $M \in \mathcal{C}$, there exists a sequence $\left\{L_{i}\right\}_{i=1}^{\infty}$ such that $L_{i} \in \mathcal{C}_{i}$ and $L_{i} \rightarrow M$. Hence there exists a positive integer $i_{0}$ such that $i>i_{0}$ implies $L_{i} \in N\left(U_{1}, \ldots, U_{n}\right) \cap C(X)$. Let $U=\cup_{i=1}^{n} U_{i}$ and let $E$ denote the component of $U$ to which $M$ belongs. Then $\left\{L_{i}\right\}_{i=i_{0}}^{\infty}$ is a sequence of continua contained in $U$ which converges to $M$. Suppose that for some $i>i_{0}, L_{i} \cap E$ $\neq \varnothing$. Then $L_{i} \subset E \subset E^{*}$ and $M \subset E^{*}$. Now $E^{*} \in N\left(V_{1}, \ldots, V_{m}\right) \cap$ $C(X)$, so it follows that there exist order arcs in $N\left(V_{1}, \ldots, V_{m}\right) \cap C(X)$ from $L_{i}$ to $E^{*}$ and from $M$ to $E^{*}$. Hence $L_{i}$ and $M$ belong to the same component of $\mathcal{U} \cap C(X)$. But $M \in \mathcal{C}$ and $L_{i} \in \mathcal{C}_{i}$ and $\mathcal{C} \cap \mathcal{C}_{i}=\varnothing$. It follows that for each $i \geqslant i_{0}, L_{i} \cap E=\varnothing$. Hence $\left\{L_{i}\right\}_{i=i_{0}}^{\infty}$ is not eventually in E.

THEOREM 3. Let $M \in C(X)$. If int $M \neq \varnothing$, then $C(X)$ is locally arcwise connected at $M$.

Proof. Let $M \in C(X)$ and let $N\left(U_{1}, \ldots, U_{n}\right) \cap C(X)$ be a basic open set containing $M$. Let $x \in$ int $M$ and let $V$ be an open set such that $x \in V \subset$ int $M$ and such that $V \subset \cap\left\{U_{i} \mid x \in U_{i}\right\}$. Then $M \in$ $N\left(U_{1}, \ldots, U_{n}, V\right) \subset N\left(U_{1}, \ldots, U_{n}\right)$. Let $L_{1}, L_{2} \in N\left(U_{1}, \ldots, U_{n}, V\right) \cap$ $C(X)$. Then $L_{1} \cap V \neq \varnothing$ and $L_{2} \cap V \neq \varnothing$, so $L_{1} \cap M \neq \varnothing$ and $L_{2} \cap M \neq$ $\varnothing$. It follows that $L_{1} \cup L_{2} \cup M \in N\left(U_{1}, \ldots, U_{n}, V\right) \cap C(X)$. Hence there exist order arcs $\mathscr{L}_{1}$ and $\mathscr{L}_{2}$ in $N\left(U_{1}, \ldots, U_{n}, V\right) \cap C(X)$ from $L_{1}$ to $L_{1} \cup L_{2}$ $\cup M$ and from $L_{2}$ to $L_{1} \cup L_{2} \cup M$. An argument similar to that used in Theorem 1 will establish that $N\left(U_{1}, \ldots, U_{n}, V\right) \cap C(X)$ contains an arc joining $L_{1}$ and $L_{2}$.

It follows from Theorem 2 of [1] and Theorem 3 that for any continuum $X$, each of $2^{X}$ and $C(X)$ is locally connected at $X$. The next two results relate indecomposability to an absence of connectedness im kleinen in $2^{X}$ and $C(X)$.

THEOREM 4. If $X$ is indecomposable, then $X$ is the only point at which $2^{X}$ is connected im kleinen.

Proof. Suppose $A \in 2^{X}, A \neq X$, and $2^{X}$ is connected im kleinen at $A$. Let $B$ be any component of $A$ and let $y \in X-A$. For each $x \in B$ let $U_{x}$ be an open set such that $y \notin U_{x^{\prime}}$. Let $\left\{U_{x_{1}}, \ldots, U_{x_{n}}\right\}$ be a finite subset of $\left\{U_{x} \mid x \in\right.$ $B\}$ such that $B \subset \cup_{i=1}^{n} U_{x_{i}}$. Then $B \in N\left(U_{x_{1}}, \ldots, U_{x_{n}}\right)$. Let $N\left(V_{1}, \ldots, V_{m}\right)$ be an open set such that

$$
B \in N\left(V_{1}, \ldots, V_{m}\right) \subset N\left(V_{1}, \ldots, V_{m}\right)^{*} \subset N\left(U_{x_{1}}, \ldots, U_{x_{n}}\right) .
$$

By Theorem 4 of [1], $2^{X}$ is connected im kleinen at $B$. Hence there exists an open set $N\left(W_{1}, \ldots, W_{p}\right)$ and a connected set $\mathcal{C}$ such that $B \in$ $N\left(W_{1}, \ldots, W_{p}\right) \subset \mathcal{C} \subset N\left(V_{1}, \ldots, V_{m}\right)$. Let $D=\left(\cup\left\{C \in 2^{X} \mid C \in \mathcal{C}\right\}\right)^{*}$. Then $D \in N\left(U_{x_{1}}, \ldots, U_{x_{n}}\right) \cap C(X), y \notin D$, and $\cup_{i=1}^{p} W_{i} \subset D$, so $D$ is a proper subcontinuum of $X$ with interior. It follows that $X$ is decomposable.

The example which follows shows that the converse of Theorem 4 is false.

EXAMPLE 1 . In Diagram 1 below, the planar continuum $X$ contains a copy 
of the Cantor fan with vertex $x_{0}$. The points $x_{2 n}\left(y_{2 n}\right)$ lie on the line segment joining $x_{0}$ and $p(q)$ and the points $x_{2 n+1}\left(y_{2 n+1}\right)$ lie on the line segment joining $x_{1}$ and $p\left(y_{1}\right.$ and $\left.q\right)$. The first coordinates of the points $x_{n}\left(y_{n}\right)$ form a strictly increasing (strictly decreasing) sequence converging to the first coordinate of $p(q)$. For each $n$ a Cantor set is chosen between $x_{n}$ and $x_{n+2}\left(y_{n}\right.$ and $\left.y_{n+2}\right)$ and a Cantor fan is constructed with vertex $x_{n+1}\left(y_{n+1}\right)$.

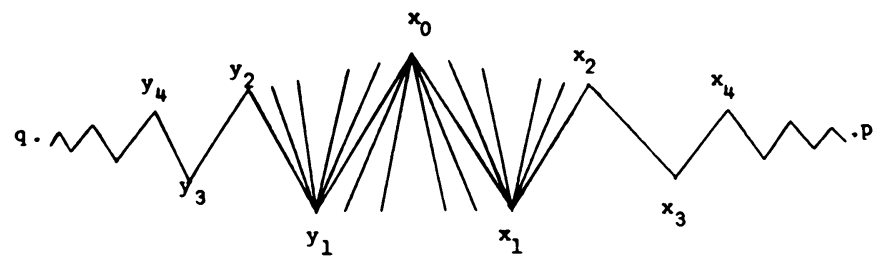

Diagram 1

$X$ is certainly decomposable, but it follows from Theorem 1 of [1] that there is no proper subcontinuum of $X$ at which $2^{X}$ is connected im kleinen. Hence by Theorem 4 of [1], $X$ is the only point at which $2^{X}$ is connected im kleinen.

THEOREM 5. If $X$ is the only point at which $C(X)$ is connected im kleinen, then $X$ is indecomposable.

Proof. If $X$ is decomposable, then $X$ has a proper subcontinuum $M$ with interior, and by Theorem 3, $C(X)$ is connected im kleinen at $M$.

The next example shows that the converse of Theorem 5 is false.
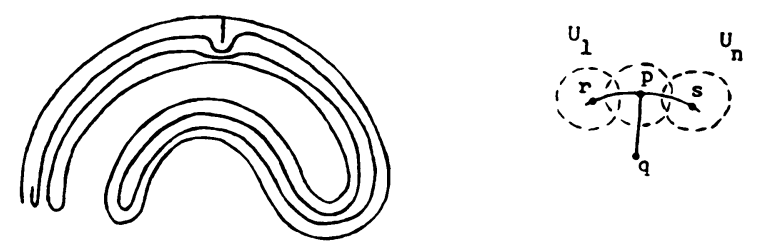

\section{Diagram 2}

Example 2. The indecomposable continuum $X$ in Diagram 2 is a slightly modified version of the indecomposable continuum commonly referred to as the "buckethandle." A small arc $[p, q]$ has been inserted at the top so that the continuum maintains the essential flavor of the "buckethandle" but contains a triod.

Let $A=[r, s]$ and let $N\left(U_{1}, \ldots, U_{n}\right) \cap C(X)$ be a basic open set as in the diagram on the right, with each $U_{i}$ chosen as a spherical open set of diameter $<\frac{1}{2}$ the distance from $[r, s]$ to $q$. If $B \in N\left(U_{1}, \ldots, U_{n}\right) \cap C(X)$, then there is a triod $C \in N\left(U_{1}, \ldots, U_{n}\right) \cap C(X)$ which contains both $A$ and $B$, and hence there is an $\operatorname{arc}$ in $N\left(U_{1}, \ldots, U_{n}\right) \cap C(X)$ joining $A$ and $B$. It follows that $C(X)$ is locally arcwise connected at the proper subcontinuum $A$. 
It follows from Corollaries 1 and 4 of [1] and Theorem 1 that if $x \in X$, then $C(X)$ is connected im kleinen at $\{x\}$ if and only if $C(X)$ is locally connected at $\{x\}$. Theorem 3 shows that the notions of connectedness im kleinen and local connectedness agree at those points $M \in C(X)$ for which int $M \neq \varnothing$. So it might be conjectured that these notions agree for all points of $C(X)$. Our final example shows that this is not the case.

EXAMPLE 3. This example is a rather extreme generalization of the example from p. 113 of [2]. We start by embedding the example just cited in the first quadrant of the $y z$ plane in $E^{3}$. Let $a(i, j)$ denote the left-hand endpoint of the $j$ th arc in the $i$ th harmonic fan and $p_{i}$ denote the vertex of the $i$ th harmonic fan. The fans are chosen so that if $a(i, j) \neq a(m, n)$, then their $z$-coordinates $z(i, j)$ and $z(m, n)$ are different. Let $M$ denote the line segment joining $\left(\frac{1}{4}, 0,0\right)$ and $\left(-\frac{1}{4}, 0,0\right)$ and let $p=(0,0,0)$ (see Diagram 3$)$.

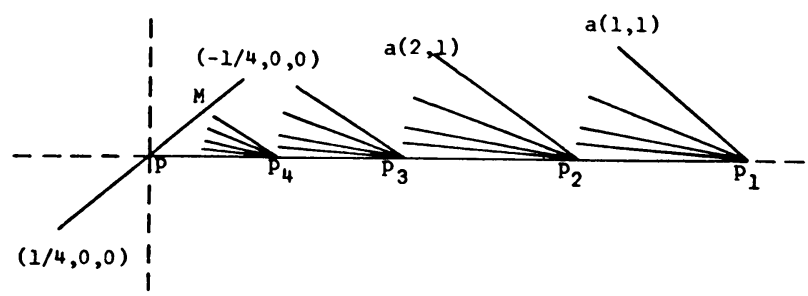

Diagram 3

Next we attach "indented" continua at each $a(i, j)$ consisting of five straight line segments in the $z(i, j)$ plane which terminate at $\left(\frac{1}{4}, 0, z(i, j)\right)$ and $\left(-\frac{1}{4}, 0, z(i, j)\right)$. Let $C(i, j)$ denote the union of the indented continuum attached at $a(i, j)$ and the arc $\left[a(i, j), p_{i}\right]$ joining $a(i, j)$ and $p_{i}$. The design is such that $\operatorname{limit}_{j \rightarrow \infty} C(i, j)=M \cup\left[p, p_{i}\right]$ (see Diagram 4). Let $Y_{1}=$ $(\cup\{C(i, j) \mid i, j \in N\}) \cup\left[p, p_{1}\right] \cup M$.

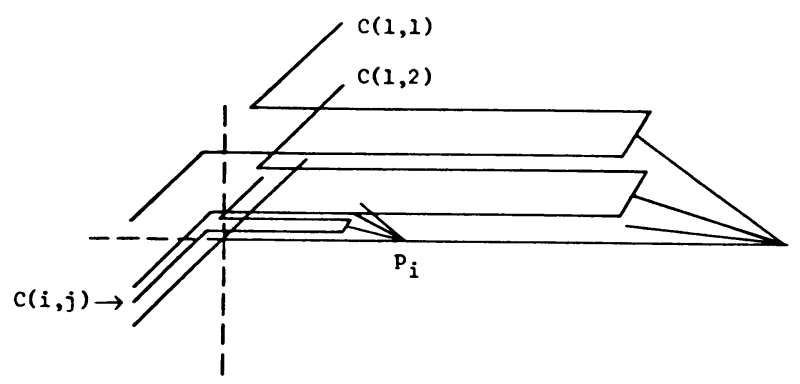

Diagram 4

We now indicate briefly why $C\left(Y_{1}\right)$ is locally arcwise connected at $M$. Let $N\left(U_{1}, \ldots, U_{n}\right) \cap C\left(Y_{1}\right)$ be a basic open set containing $M$ such that $\left\{U_{1}, \ldots, U_{n}\right\}$ consists of a simple chain of open spheres of diameter $<\frac{1}{8}$ from $\left(\frac{1}{4}, 0,0\right)$ to $\left(-\frac{1}{4}, 0,0\right)$. Let $i_{0}=\inf \left\{i \in N \mid p_{i} \in \cup_{j=1}^{n} U_{j}\right\}$. It is apparent that if $D \in N\left(U_{1}, \ldots, U_{n}\right) \cap C\left(Y_{1}\right)$ and there does not exist an arc in $N\left(U_{1}, \ldots, U_{n}\right) \cap C\left(Y_{1}\right)$ joining $M$ and $D$, then for some $j_{1}$, 


$$
D \subset C\left(i_{0}-1, j_{1}\right) \text {. }
$$

Let $C\left(i_{0}, j_{2}\right) \in N\left(U_{1}, \ldots, U_{n}\right) \cap C\left(Y_{1}\right)$. Choose "small" arcwise connected open sets $N\left(V_{1}, \ldots, V_{m}\right) \cap C\left(Y_{1}\right)$ and $N\left(W_{1}, \ldots, W_{p}\right) \cap C\left(Y_{1}\right)$ containing $D$ and $C\left(i_{0}, j_{2}\right)$, respectively, and let $O$ be a "small" open set containing $\left[a\left(i_{0}-1, j_{1}\right), p_{i_{0}-1}\right] \cup\left[p_{i_{0}-1}, p_{i_{0}}\right]$. Then

$$
\begin{aligned}
\left\{N\left(V_{1}, \ldots, V_{m}, O \cup\left(\bigcup_{i=1}^{p} W_{i}\right)\right)\right. & \left.\cup N\left(W_{1}, \ldots, W_{p}, O \cup\left(\bigcup_{i=1}^{m} V_{i}\right)\right)\right\} \\
& \cap C\left(Y_{1}\right)
\end{aligned}
$$

is an arcwise connected open set containing $D$ and $C\left(i_{0}, j_{2}\right)$. It follows that we can "enlarge" $N\left(U_{1}, \ldots, U_{n}\right) \cap C\left(Y_{1}\right)$ to an arcwise connected open set $\checkmark$ such that for some $p^{1} \in\left(p_{i_{0}-2}, p_{i_{0}-1}\right)$,

$$
\cup \mathscr{V} \subset M \cup\left[p, p^{1}\right) \cup\left(\cup\left\{C(i, j) \mid i>i_{0}-1, j \in N\right\}\right) .
$$

The purpose of the inductive process which follows is to introduce enough points of nonconnectedness im kleinen to prevent the resulting hyperspace from being locally connected at $M$.

Next we construct a sequence $C\left(2,1, j^{1}\right)$ of "indented" continua so that $\operatorname{limit}_{j^{1} \rightarrow \infty} C\left(2,1, j^{1}\right)=C(2,1) \cup\left[p_{1}, p_{2}\right]$ and such that $\left(\cup\left\{C\left(2,1, j^{1}\right) \mid j^{1} \in\right.\right.$ $N\}) \cap Y_{1}=\left\{p_{1}\right\}$. Further, if $j_{1}^{1} \neq j_{2}^{1}$, we require that $C\left(2,1, j_{1}^{1}\right) \cap$ $C\left(2,1, j_{2}^{1}\right)=\left\{p_{1}\right\}$. Inductively, for each positive integer $j$ we construct a sequence $C\left(2, j, j^{1}\right)$ so that $\operatorname{limit}_{j^{\prime} \rightarrow \infty} C\left(2, j, j^{1}\right)=C(2, j) \cup\left[p_{1}, p_{2}\right]$. The sequences are chosen so that $\left(\cup\left\{C\left(2, j, j^{1}\right) \mid j, j^{1} \in N\right\}\right) \cap Y_{1}=\left\{p_{1}\right\}$ and such that if $j_{1} \neq j_{2}$ or $j_{1}^{1} \neq j_{2}^{1}$, then $C\left(2, j_{1}, j_{1}^{1}\right) \cap C\left(2, j_{2}, j_{2}^{1}\right)=\left\{p_{1}\right\}$ (see Diagram 5). Let $Y_{2}=Y_{1} \cup\left(\cup\left\{C\left(2, j, j^{1}\right) \mid j, j^{1} \in N\right\}\right)$.

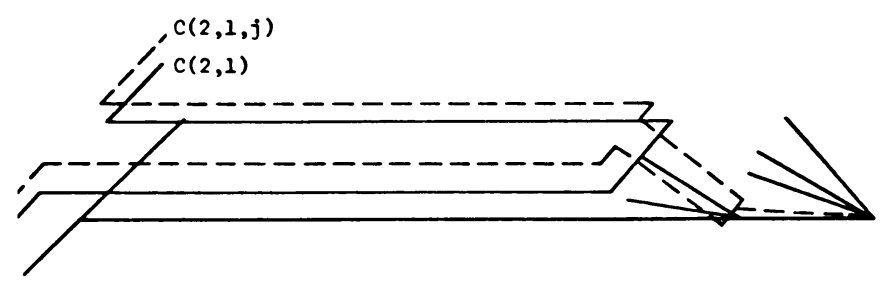

\section{Diagram 5}

We now proceed to define $Y_{i}$ inductively. For each positive integer $j$ we construct a sequence $C\left(i, j, j^{1}\right)$ such that

$$
\operatorname{limit}_{j^{1} \rightarrow \infty} C\left(i, j, j^{1}\right)=C(i, j) \cup\left[p_{i-1}, p_{i}\right]
$$

such that

$$
\left(\cup\left\{C\left(i, j, j^{1}\right) \mid j, j^{1} \in N\right\}\right) \cap Y_{i-1}=\left\{p_{i-1}\right\},
$$

and such that if $j_{1} \neq j_{2}$ or $j_{1}^{1} \neq j_{2}^{1}$, then $C\left(i, j_{1} j_{1}^{1}\right) \cap C\left(i, j_{2}, j_{2}^{1}\right)=\left\{p_{i-1}\right\}$. 
Inductively, for each $k=1, \ldots, i-2$ and for each $j, j^{1}, \ldots, j^{k}$, we construct a sequence $C\left(i, j, j^{1}, \ldots, j^{k}, j^{k+1}\right)$ so that

$$
\begin{aligned}
\operatorname{limit}_{j^{k+1} \rightarrow \infty} C\left(i, j, j^{1}, \ldots, j^{k}, j^{k+1}\right) & \\
& =C\left(i, j, j^{1}, \ldots, j^{k}\right) \cup\left[p_{i-(k+1)}, p_{i-k}\right]
\end{aligned}
$$

and such that

$$
\begin{aligned}
& \left(\cup\left\{C\left(i, j, j^{1}, \ldots, j^{k}, j^{k+1}\right) \mid j, j^{1}, \ldots, j^{k}, j^{k+1} \in N\right\}\right) \\
& \cap\left(Y_{i-1} \cup\left(\cup\left\{C\left(i, j, j^{1}, \ldots, j^{k}\right) \mid j, j^{1}, \ldots, j^{k} \in N\right\}\right)\right)=\left\{p_{i-(k+1)}\right\} .
\end{aligned}
$$

Further we require that if $j_{1} \neq j_{2}$ or if for at least one $i \in\{1, \ldots, k+1\}, j_{1}^{i}$ $\neq j_{2}^{i}$, then

$$
C\left(i, j_{1}, j_{1}^{1}, \ldots, j_{1}^{k}, j_{1}^{k+1}\right) \cap C\left(i, j_{2}, j_{2}^{1}, \ldots, j_{2}^{k}, j_{2}^{k+1}\right)=\left\{p_{i-(k+1)}\right\}
$$

(see Diagram 6). Now we let

$$
\begin{aligned}
Y_{i}= & Y_{i-1} \cup\left(\cup\left\{C\left(i, j, j^{1}\right) \mid j, j^{1} \in N\right\}\right) \\
& \cup\left(\cup\left\{C\left(i, j, j^{1}, j^{2}\right) \mid j, j^{1}, j^{2} \in N\right\}\right) \cup \cdots \\
& \cup\left(\cup\left\{C\left(i, j, j^{1}, \ldots, j^{i-1}\right) \mid j, j^{1}, \ldots, j^{i-1} \in N\right\}\right) .
\end{aligned}
$$

Finally we let $X=\cup_{i=1}^{\infty} Y_{i}$.

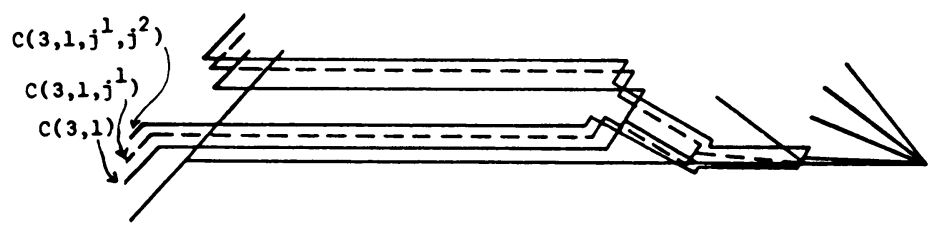

\section{DiAgRAM 6}

It follows from Theorem 2 that $C(X)$ is connected im kleinen at $\boldsymbol{M}$. Suppose $N\left(U_{1}, \ldots, U_{n}\right) \cap C(X)$ is the type of open set described after the first stage of the construction. If $\mathfrak{V}$ is a connected open set and $M \in \mathcal{V} \subset$ $N\left(U_{1}, \ldots, U_{n}\right) \cap C(X)$, then for some $i \in N, M \cup\left[p, p_{i}\right] \in \mathcal{V}$. So there exists a sequence $\left\{B_{j}\right\}_{j=1}^{\infty}$ such that $B_{j} \rightarrow M \cup\left[p, p_{i}\right]$ and such that for each $j \in N, B_{j} \subset C(i-1, j)$. Now any connected subset of $N\left(U_{1}, \ldots, U_{n}\right) \cap$ $C(X)$ containing $B_{j}$ and $M$ must contain a continuum $D(i-1, j) \subset$ $C(i-1, j)$ such that $p_{i-1} \in D(i-1, j)$. Thus for some $j_{1}$ there exists a sequence $\left\{B\left(j_{1}, j^{1}\right)\right\}_{j^{1}=1}^{\infty}$ such that $B\left(j_{1}, j^{1}\right) \rightarrow D\left(i-1, j_{1}\right)$, and such that for each $j^{1} \in N, B\left(j_{1}, j^{1}\right) \subset C\left(i-1, j_{1}, j^{1}\right)$. It follows that $\mathfrak{V}$ must contain a continuum $D\left(i-1, j_{1}, j_{1}^{1}\right) \subset C\left(i-1, j_{1}, j_{1}^{1}\right)$ such that $P_{i-2} \in D(i-$ $\left.1, j_{1}, j_{1}^{l}\right)$. Continuing this process, after $i-1$ steps we obtain $p_{1} \in \cup \mathfrak{V}$. Hence $C(X)$ is not locally connected at $M$. 


\section{REFERENCES}

1. J. T. Goodykoontz, Jr., Connectedness im kleinen and local connectedness in $2^{X}$ and $C(X)$, Pacific J. Math. 53 (1974), 387-397.

2. J. G. Hocking and G. S. Young, Topology, Addison-Wesley, Reading, Mass., 1961.

3. J. L. Kelley, Hyperspaces of a continuum, Trans. Amer. Math. Soc. 52 (1942), 23-36.

4. E. Michael, Topologies on spaces of subsets, Trans. Amer. Math. Soc. 71 (1951), 152-182.

5. G. T. Whyburn, Analytical topology, Amer. Math. Soc. Colloq. Publ., vol. 28, Amer. Math. Soc., Providence, R.I., 1942.

Department of Mathematics, West Virginia University, Morgantown, West Virginia 26506 